\title{
Efeito de Diferentes Níveis de Sódio e Fósforo sobre o Desempenho e a Qualidade da Casca dos Ovos de Poedeiras Comerciais ${ }^{1}$
}

\author{
Douglas Emygdio de Faria², Otto Mack Junqueira ${ }^{3}$, Nilva Kazue Sakomura ${ }^{3}$, \\ Áureo Evangelista Santana ${ }^{3}$
}

\begin{abstract}
RESUMO - Conduziu-se um experimento com o objetivo de verificar o efeito de diferentes níveis de sódio e fósforo (P) sobre o desempenho e a qualidade da casca dos ovos e de determinados constituintes sangüíneos, em 144 galinhas Hy-Line W36, com 60 semanas de idade, distribuídas em 36 unidades experimentais com quatro aves cada. O delineamento utilizado foi o de blocos ao acaso em arranjo fatorial (3 x 3), níveis de sódio $(0,16 ; 0,20$; e $0,24 \%)$ e níveis de fósforo total $(0,35 ; 0,45$; e $0,55 \%)$, totalizando nove tratamentos, dois blocos com duas repetições em cada bloco. As rações experimentais foram isoprotéicas (16,0\% de PB), isocalóricas (2800 kcal de EM/kg) e isocálcicas $(4,0 \%$ de $\mathrm{Ca})$. Consumo de ração, produção e massa de ovos, conversão alimentar, porcentagem e espessura da casca e densidade aparente dos ovos não foram alteradas pelos níveis de sódio. Entretanto, quando as galinhas foram alimentadas com dietas contendo $0,35 \%$ de $\mathrm{P}$ total, verificou-se comprometimento da produção e massa de ovos e conversão alimentar, sem alteração das características de qualidade da casca. Houve interação entre os fatores para o peso dos ovos. Baixos níveis de P total não foram efetivos em melhorar a qualidade da casca dos ovos, mas prejudicaram o desempenho das galinhas.
\end{abstract}

Palavras-chave: fósforo, nutrição, poedeiras, qualidade da casca dos ovos, sódio

\section{Effect of Different Levels of Sodium and Phosphorus on Performance and Eggshell Quality of Laying Hens}

\begin{abstract}
An experiment was carried out to verify the effect of different levels of sodium and phosphorus (P) on the performance, eggshell quality and on some blood constituents of 144 laying hens Hy-Line W36, aged 60 weeks, allotted to 36 experimental units with four hens each. A randomized experimental block design was used in a factorial arrangement ( $3 \mathrm{x} 3)$, sodium levels (.16, .20 and .24\%) and phosphorus levels $(.35, .45$ and $.55 \%)$, totalizing nine treatments, two blocks with two replicates each. The experimental diets were isoproteic $(16.0 \% \mathrm{CP})$, isoenergetic $(2,800 \mathrm{kcal} \mathrm{ME} / \mathrm{kg})$ and isocalcium $(4.0 \% \mathrm{Ca})$. Feed intake, egg production, egg mass, feed:gain ratio, shell percentage, shell thickness and egg specific gravity were not affected by sodium levels. However, when the hens were fed diets containing . $35 \%$ total $\mathrm{P}$, there was impairment of the egg production, egg mass and feed:gain ratio, without modification of the eggshell quality characteristics. There was interaction between the factors for egg weight. Low phosphorus levels were not effective to improve the eggshell quality, but impaired hens performance.
\end{abstract}

Key Words: eggshell quality, laying hens, nutrition, phosphorus, sodium

\section{Introdução}

Segundo SCOTT et al. (1982), o sódio é considerado o principal cátion monovalente do fluido extracelular. Todos os organismos vivos, animal ou vegetal, necessitam deste elemento para o seu metabolismo normal. As recomendações de sódio para poedeiras podem variar segundo a fonte de consulta: 0,12\%; 165 e $150 \mathrm{mg} / \mathrm{ave} / \mathrm{dia} ; 145 \mathrm{mg} / \mathrm{ave} / \mathrm{dia} ;$ e 0,17\%, conforme SCOTT et al. (1982), NRC (1984, 1994), ROSTAGNO et al. (1985) e LEESON e SUMMERS (1991), respectivamente.
Com a proposta de avaliar a exigência de sódio dietético em poedeiras, REID (1977) utilizou os níveis de 0,$04 ; 0,10 ; 0,15 ; 0,19 ; 0,21 ; 0,22 ;$ e $0,37 \%$ de sódio, constatando significativo comprometimento da produção de ovos e conversão alimentar com a administração do menor nível $(0,04 \%)$. Esse autor concluiu, por intermédio de análise de regressão, que a estimativa das exigências de sódio estava ao redor de $0,126 \%$ da dieta, correspondedo à ingestão de $141 \mathrm{mg}$ de sódio/ave/dia.

LEESON e SUMMERS (1979) comentaram sobre a possibilidade de melhoria da qualidade da casca

\footnotetext{
${ }^{1}$ Parte da Tese do primeiro autor apresentada à Faculdadde de Ciências Agrárias e Veterinárias (FCAV/UNESP), Jaboticabal, SP, para obtenção do grau de Doutor em Zootecnia.

${ }^{2}$ Docente do Dep. de Zootecnia da Faculdade de Zootecnia e Engenharia de Alimentos (FZEA/USP), Campus de Pirassununga, SP. E.mail: defaria@usp.br

${ }^{3}$ Docente da FCAV/UNESP, Campus de Jaboticabal, SP.
} 
por intermédio do incremento do nível de sódio dietético acima de $0,20 \%$ para galinhas alimentadas com dietas à base de milho e soja. Nesse sentido, esses autores desenvolveram um estudo utilizando dois níveis de fósforo disponível $(0,36$ e $0,56 \%)$ e duas fontes de cálcio (calcário e farinha de casca de ostras). Os autores verificaram que não houve efeito significativo dos tratamentos sobre o consumo de ração e o peso corporal final, sendo que a deformação da casca foi mais pronunciada com a utilização dos níveis de $0,56 \%$ de fósforo disponível com $0,25 \%$ de sódio, em que o calcário foi a principal fonte de cálcio. Essa resposta não foi verificada quando se utilizou farinha de casca de ostras. Concluiu-se que, sob condições ambientais controladas com temperatura ao redor de $20^{\circ} \mathrm{C}$, não se detectaram vantagens em utilizar níveis mais elevados de sódio na dieta e o nível de $0,15 \%$ de sódio seria adequado para suportar desempenho satisfatório.

CHOI et al. (1980) testaram três níveis de cloreto de sódio $(0 ; 0,35$; e $1,40 \%)$, o que correspondeu aos níveis $0 ; 0,15$; e $0,55 \%$ de sódio, com três níveis de fósforo total $(0,30 ; 0,75 ; \mathrm{e} 1,40 \%)$ nas dietas de poedeiras com 69 semanas de idade. Na análise global do experimento, CHOI et al. (1980) não encontraram diferenças significativas na produção de ovos de galinhas alimentadas com as dietas contendo os diferentes níveis de fósforo. No entanto, a produção de ovos foi inferior, quando não houve a adição de cloreto de sódio nas dietas em comparação aos outros dois níveis de suplementação, que não diferiram entre si.

DAMRON e HARMS (1980) conduziram dois experimentos com poedeiras na fase inicial de produção para estudar os efeitos interativos de diferentes níveis de cálcio $(2,25 ; 3,6$; e 6,0\%), fósforo total $(0,45$ e $0,70 \%)$ e cloreto de sódio $(0,18 ; 0,35$; e $0,70 \%)$, que corresponderam aos níveis de 0,$07 ; 0,14 ;$ e $0,27 \%$ de sódio. Quando se analisaram os resultados obtidos com a utilização de diferentes níveis de cloreto de sódio e cálcio, esses autores constataram que a produção aumentou significativamente, à medida que se elevou a inclusão de cloreto de sódio na dieta, enquanto, para os níveis de cálcio, o maior valor para produção foi verificado com o nível de 3,5\% de cálcio. O peso dos ovos não foi alterado pelos tratamentos e o consumo de ração não diferiu entre os níveis de sal, mas foi reduzido com o aumento do nível de cálcio. Os piores índices de conversão alimentar foram obtidos com o nível de $2,25 \%$ de cálcio e $0,18 \%$ de sal na ração. Com a associação dos níveis de sal e de fósforo, os autores não verificaram efeitos significa- tivos para o consumo de ração e peso dos ovos. Entre os níveis de fósforo total, não houve diferenças na produção de ovos e conversão alimentar, sendo que essas características foram prejudicadas quando as galinhas receberam as dietas contendo $0,18 \%$ de sal ou o equivalente a aproximadamente $0,07 \%$ de sódio.

Em um dos experimentos conduzidos por JUNQUEIRA et al. (1984), foram avaliados três níveis de fósforo total $(0,2 ; 0,6 ;$ e $1,4 \%)$ e quatro níveis de sódio $(0 ; 0,06 ; 0,22$; e $0,45 \%)$ fornecidos pelo bicarbonato de sódio. Em todos os níveis de fósforo, observou-se que o fósforo plasmático se comportou de maneira cíclica, diminuindo ligeiramente da oviposição até 6 horas após e, em seguida, alcançando um pico 21 horas após a oviposição. $\mathrm{OpH}$ sangüíneo não foi alterado pelos níveis de fósforo, sódio e cloro dietéticos, porém verificou-se que o excesso de bases, bicarbonato e $\mathrm{CO}_{2}$ total sangüíneos reduziu, à medida que se aumentou o conteúdo de fósforo da dieta, enquanto o nível de fósforo sangüíneo foi reduzido, à medida que se elevou o nível de bicarbonato de sódio. Entre os níveis de sódio, não se verificou efeito sobre a produção e o peso dos ovos e a conversão alimentar, no entanto, a densidade aparente foi superior, quando se utilizaram os níveis de 0,06 e $0,22 \%$ de sódio, principalmente quando a dieta continha $1,4 \%$ de fósforo total. Os autores comentaram sobre as possíveis evidências de que o sódio pode funcionar como removedor do excesso de fósforo sangüíneo, resultando em melhor qualidade da casca dos ovos.

JACKSON et al. (1987) conduziram três experimentos com diferentes manipulações de nutrientes, visando examinar a relação entre peso do ovo e resistência da casca. Um dos referidos estudos avaliou três níveis de sódio $(0,15 ; 0,30$; e $0,45 \%)$ e três níveis de fósforo disponível $(0,25 ; 0,45 ; \mathrm{e} 0,65 \%)$. Os autores não observaram interação entre os fatores estudados para o peso dos ovos, mas verificaram que houve redução, à medida que se aumentou o nível de sódio dietético de 0,15 para $0,45 \%$. Verificou-se interação significativa para a produção de ovos, quando as galinhas foram alimentadas com as dietas contendo $0,45 \%$ de sódio e $0,25 \%$ de fósforo disponível, resultando em decréscimo na taxa de produção após 24 semanas de idade. Os resultados obtidos para resistência da casca não apresentaram tendência consistente para o efeito dos níveis de sódio, porém, ao diminuir o nível de fósforo de 0,65 para $0,25 \%$ da dieta, verificou-se aumento dos valores de resistência da casca.

JUNQUEIRA (1988) avaliou níveis e fontes de sódio para poedeiras com 48 semanas de idade, 
constituindo quatro tratamentos. Os dois primeiros tratamentos consistiram da adição de 0,37 e $0,67 \%$ de cloreto de sódio, respectivamente. No terceiro e quarto tratamentos, incluiu-se a adição de $0,41 \%$ de bicarbonato de sódio e $0,34 \%$ de sulfato de sódio a uma dieta que continha $0,37 \%$ de $\mathrm{NaCl}$. No primeiro tratamento, o nível de sódio foi de $0,17 \%$ e no restante, de $0,28 \%$. Não se constataram diferenças significativas nas características de produção, peso e massa de ovos, consumo de ração e conversão alimentar. A densidade aparente do ovo foi melhorada, quando se utilizou o nível de $0,28 \%$ de sódio, independente da fonte de sódio e das relações sódio:cloro $(0,61: 1$ ou $1,08: 1)$. Os menores valores de $\mathrm{pH}$ para os constituintes sangüíneos foram obtidos quando as aves receberam as dietas contendo $0,67 \%$ de $\mathrm{NaCl}$ e $0,37 \%$ de $\mathrm{NaCl}$ mais $0,34 \%$ de $\mathrm{NaSO}_{4}$, enquanto o maior valor foi obtido quando se adicionou $0,41 \%$ de $\mathrm{NaHCO}_{3}$ à dieta contendo $0,37 \%$ de $\mathrm{NaCl}$. De maneira geral, o maior valor obtido para $\mathrm{pH}$ está associado também a maiores valores obtidos para bicarbonato e $\mathrm{pCO}_{2}$.

Com relação ao fósforo, verifica-se também certa variação nas recomendações dos níveis de suplementação nas dietas de poedeiras. Dessa forma, os níveis sugeridos são $0,35 \%$ e 350, 250, 385 e $400 \mathrm{mg} / \mathrm{ave} /$ dia de fósforo disponível, segundo SCOTT et al. (1982), NRC $(1984,1994)$, ROSTAGNO et al. (1985) e LEESON e SUMMERS (1991), respectivamente. Algumas recomendações são realizadas segundo a faixa de idade das poedeiras; HARMS (1982) sugere ingestão diária de 650, 550 e 450 mg de fósforo total para as respectivas faixas etárias de 20 a 36, 37 a 52 e após 53 semanas, ao passo que ROLAND (1986), de maneira similar, recomenda 700,600 e $500 \mathrm{mg} / \mathrm{ave} / \mathrm{dia}$ de fósforo total para as mesmas faixas etárias.

O desempenho de poedeiras tem sido prejudicado por baixos (SUMMERS et al., 1976; HOLDER, 1981; MILES et al., 1983; B AR e HURWITZ, 1984; JUNQUEIRA, 1988; e LEESON et al., 1993) ou altos níveis de fósforo dietético (MILES et al., 1983; JUNQUEIRA, 1988). Por outro lado, a qualidade da casca dos ovos também pode ser prejudicada por altos (OSTERHOUT, 1980; HARMS, 1982; MILES et al., 1983; SAID e SULLIVAN, 1985; JUNQUEIRA, 1988; SAKOMURA et al., 1993; e LEESON et al., 1993) ou baixos níveis de fósforo dietético (JUNQUEIRA, 1988; LEESON et al., 1993). Contudo, há referências de melhoria da qualidade da casca dos ovos, quando se empregam baixos níveis de fósforo (HOLDER, 1981).

ROLAND (1986) estabeleceu comentários sobre as possíveis hipóteses envolvidas com a melhoria da qualidade da casca dos ovos, quando da redução dos níveis de fósforo, mas ampla revisão envolvendo vários estudos com este elemento foi realizada por ROLAND (1989).

SCHEIDELER e AL-BATSHAN (1994) argumentaram sobre os possíveis benefícios decorrentes da administração de baixos níveis de fósforo nas dietas de poedeiras, porém questionaram sobre o período de tempo que as aves suportariam esses baixos níveis sem apresentar osteoporose. Entretanto, ROLAND e GORDON (1996) reconhecem a confusão proporcionada pelo fato de baixos níveis de fósforo induzirem melhoria na qualidade da casca dos ovos, sendo que algumas ponderações são realizadas em função desse tipo de resposta ser temporária e, também, por existir variação entre as aves na resposta à dieta deficiente em fósforo.

O objetivo do presente estudo foi avaliar o efeito de diferentes níveis de sódio e fósforo sobre as características de desempenho e a qualidade da casca dos ovos e de determinados constituintes sangüíneos em poedeiras na fase final de produção.

\section{Material e Métodos}

O experimento foi realizado no aviário experimental da Faculdade de Ciências Agrárias e Veterinárias (FCAV/UNESP), Jaboticabal, com duração de oito períodos de uma semana cada. Foram utilizadas 144 galinhas Hy-Line W36 com 60 semanas de idade, distribuídas em 36 parcelas, sendo cada unidade experimental composta por quatro aves.

$\mathrm{O}$ delineamento experimental utilizado foi o de blocos ao acaso em arranjo fatorial $3 \times 3$, com os fatores: níveis de sódio $(0,16 ; 0,20 ;$ e $0,24 \%)$ e níveis de fósforo total $(0,35 ; 0,45$; e $0,55 \%)$, totalizando nove tratamentos, dois blocos e duas repetições por bloco. Os blocos foram constituídos em função do peso corporal das aves, conforme as seguintes faixas de peso: 1,350 a $1,645 \mathrm{~kg}$ e 1,650 a $1,900 \mathrm{~kg}$.

Foram usadas rações à base de milho e farelo de soja, isoprotéicas (16,0\% de PB), isocalóricas (2800 $\mathrm{kcal}$ de $\mathrm{EM} / \mathrm{kg}$ ) e isocálcicas $(4,0 \%$ de $\mathrm{Ca}$ ), formuladas conforme as exigências nutricionais por ave por dia e o consumo diário de $105 \mathrm{~g} / \mathrm{ave} / \mathrm{dia}$, segundo ROSTAGNO et al. (1985). O nível de sódio considerado para o sal comum foi de $39,74 \%$ (ROSTAGNO et al., 1985), correspondendo, para cada acréscimo 
FARIA et al.

de $0,1 \%$ de sal comum nas rações experimentais, acréscimo de $0,04 \%$ no nível de sódio. A composição percentual e os níveis nutricionais calculados das rações experimentais encontram-se na Tabela 1 .

As características de desempenho avaliadas foram consumo de ração (g/ave/dia), produção de ovos (\% ovos/ave/dia), peso dos ovos (g), conversão alimentar (kg ração/kg ovo) e massa de ovos (g/ave/ dia). A produção de ovos foi registrada diariamente e o cálculo, realizado no final de cada período experimental de uma semana. O peso médio dos ovos foi obtido por intermédio da pesagem de todos os ovos produzidos nos últimos dois dias de cada período experimental. A massa de ovos é o resultado da multiplicação da produção pelo peso médio dos ovos. As características de qualidade da casca dos ovos consideradas foram porcentagem de casca, espessura da casca $(\mathrm{mm})$ e densidade aparente do ovo $(\mathrm{g} / \mathrm{mL}$ $\mathrm{H}_{2} \mathrm{O}$ ). As soluções salinas utilizadas para obtenção da densidade aparente dos ovos foram preparadas conforme recomendações de MORENG e AVENS (1990), com os devidos ajustes para um volume de 20 litros de água. A faixa de densidade das soluções foi de 1,0650 a 1,0950 com intervalos de 0,0025.

No final do experimento, foram coletadas amostras de sangue após uma hora da postura, de uma galinha por unidade experimental, por meio de punção intracardíaca. Analisaram-se o pH sangüíneo e as pressões parciais de oxigênio $\left(\mathrm{pO}_{2}-\mathrm{mm} \mathrm{Hg}\right)$ e de dióxido de carbono $\left(\mathrm{pCO}_{2}-\mathrm{mm} \mathrm{Hg}\right.$ ). Também foram determinadas a contagem total de eritrócitos (células $/ \mathrm{mm}^{3}$ ) e leucócitos (células $/ \mathrm{mm}^{3}$ ), a concentração de hemoglobina $(\mathrm{g} / 100 \mathrm{~mL})$, inclusive em amostra centrifugada a 1500 rotações por minuto (rpm) por cinco minutos, e o hematócrito (\%), segundo JAIN (1986).

Os dados obtidos foram analisados por intermédio do sistema SAS (1988) e as médias dos tratamentos, comparadas pelo teste de Tukey a $5 \%$ de probabilidade.

Tabela 1 - Composição percentual e níveis nutricionais das dietas experimentais

Table 1 - Percentage composition and nutritional levels of the experimental diets

\begin{tabular}{|c|c|c|c|}
\hline \multirow[t]{2}{*}{$\begin{array}{l}\text { Ingredientes } \\
\text { Ingredients }\end{array}$} & \multicolumn{3}{|c|}{$\begin{array}{c}\text { Rações experimentais } \\
\text { Experimental diets }\end{array}$} \\
\hline & 1 & 2 & 3 \\
\hline Milho moído (Ground corn) & 64,68 & 65,11 & 65,55 \\
\hline Farelo de soja (Soybean meal) & 22,61 & 22,52 & 22,44 \\
\hline Calcário (Limestone) & 9,44 & 9,80 & 10,15 \\
\hline Fosfato bicálcico (Dicalcium phosphate) & 1,33 & 0,77 & 0,21 \\
\hline Óleo de soja (Soybean oil) & 0,85 & 0,70 & 0,55 \\
\hline Sal (Salt) & 0,32 & 0,32 & 0,32 \\
\hline Suplemento vitamínico mineral e aditivos ${ }^{1}$ & 0,50 & 0,50 & 0,50 \\
\hline \multicolumn{4}{|l|}{ Vitamin mineral and additives premix } \\
\hline Porção variável ${ }^{2}$ (Variable portion) & 0,27 & 0,28 & 0,28 \\
\hline \multicolumn{4}{|l|}{ Níveis nutricionais calculados } \\
\hline \multicolumn{4}{|l|}{ Calculated nutritional levels } \\
\hline Energia metabolizável (kcal/kg) & 2800 & 2800 & 2800 \\
\hline \multicolumn{4}{|l|}{ Metabolizable energy } \\
\hline Proteína bruta (Crude protein), $\%$ & 16,00 & 16,00 & 16,00 \\
\hline Cálcio (Calcium), \% & 4,00 & 4,00 & 4,00 \\
\hline Sódio (Sodium), \% & 0,16 & 0,16 & 0,16 \\
\hline Fósforo total (Total phosphorus), \% & 0,55 & 0,45 & 0,35 \\
\hline Fósforo disponível (Available phosphorus), \% & 0,34 & 0,24 & 0,14 \\
\hline \multicolumn{4}{|c|}{ 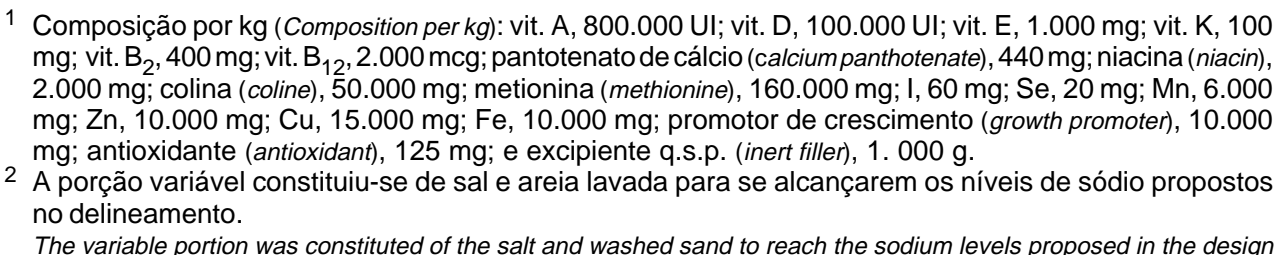 } \\
\hline
\end{tabular}




\section{Resultados e Discussão}

Os resultados das características consumo de ração, produção e peso dos ovos encontram-se na Tabela 2. Na maioria dos períodos, inclusive no período experimental total, o consumo de ração não foi influenciado pelos níveis de sódio e fósforo. $\mathrm{Da}$ mesma forma, a produção de ovos não foi afetada pelos níveis de sódio. No entanto, constatou-se decréscimo dessa característica no terceiro e quarto períodos experimentais, quando as galinhas receberam as dietas com $0,35 \%$ de fósforo total. $\mathrm{Na}$ análise do período experimental total, verificou-se que o nível de $0,35 \%$ proporcionou menor taxa de produção de ovos em relação ao nível de $0,55 \%$ de fósforo total.

O peso dos ovos foi reduzido com a utilização do nível de $0,35 \%$ de fósforo total em relação ao maior nível adotado neste estudo. Interações significativas entre os fatores foram observadas no terceiro, quinto, sétimo e oitavo períodos experimentais. Considerando o período experimental total, verificou-se também interação entre os fatores, cujo desdobramento dos tratamentos (não mostrado) indicou a ocorrência de menores valores para o peso dos ovos, quando houve

Tabela 2 - Consumo de ração, produção de ovos e peso dos ovos, em cada período de sete dias e no período experimental total, de galinhas alimentadas com diferentes níveis dietéticos de sódio e fósforo

Table 2 - Feed intake, egg production and egg weight in each seven days period and in the total experimental period, of the hens fed different dietary levels of sodium and phosphorus

\begin{tabular}{|c|c|c|c|c|c|c|}
\hline \multirow[t]{2}{*}{$\begin{array}{l}\text { Item/Período } \\
\text { Period }\end{array}$} & \multicolumn{3}{|c|}{$\begin{array}{l}\text { Níveis de sódio (\%) } \\
\text { Sodium levels }\end{array}$} & \multicolumn{3}{|c|}{$\begin{array}{l}\text { Níveis de fósforo total } \\
\text { Total phosphorus levels }\end{array}$} \\
\hline & 0,16 & 0,20 & 0,24 & 0,35 & 0,45 & 0,55 \\
\hline $\begin{array}{l}\text { Consumo de ração } \\
\text { Feed intake }\end{array}$ & & & $\begin{array}{l}\text { ve/dia } \\
\text { en/day }\end{array}$ & & & \\
\hline $\begin{array}{l}1 \\
2 \\
3 \\
4 \\
5 \\
6 \\
7 \\
8 \\
\text { Total }\end{array}$ & $\begin{array}{c}98,33 \\
103,63^{\mathrm{ab}} \\
95,24 \\
107,98 \\
105,57 \\
103,43 \\
102,44 \\
98,81 \\
101,72\end{array}$ & $\begin{array}{c}95,42 \\
105,48^{\mathrm{a}} \\
97,02 \\
108,36 \\
108,48 \\
107,02 \\
104,47 \\
98,87 \\
103,14\end{array}$ & $\begin{array}{c}95,78 \\
96,73^{\mathrm{b}} \\
91,61 \\
103,22 \\
107,00 \\
101,90 \\
99,58 \\
97,32 \\
99,40\end{array}$ & $\begin{array}{c}95,18 \\
97,50 \\
87,92^{b} \\
101,37 \\
103,33 \\
101,07 \\
100,84 \\
96,01 \\
97,90\end{array}$ & $\begin{array}{c}96,67 \\
104,47 \\
96,37 \mathrm{a} \\
108,51 \\
109,14 \\
105,98 \\
103,87 \\
99,88 \\
103,11\end{array}$ & $\begin{array}{c}97,68 \\
103,87 \\
99,58^{\mathrm{a}} \\
109,67 \\
108,57 \\
105,68 \\
101,79 \\
99,11 \\
103,25\end{array}$ \\
\hline $\begin{array}{l}\text { Produção de ovos } \\
\text { Egg production }\end{array}$ & & & $\begin{array}{l}\text { s/ave/di } \\
\text { /hen/day }\end{array}$ & & & \\
\hline $\begin{array}{l}1 \\
2 \\
3 \\
4 \\
5 \\
6 \\
7 \\
8 \\
\text { Total }\end{array}$ & $\begin{array}{l}76,19 \\
77,68 \\
76,79 \\
77,68 \\
77,68 \\
76,67 \\
73,21 \\
75,30 \\
76,53\end{array}$ & $\begin{array}{l}73,21 \\
75,00 \\
72,32 \\
77,68 \\
75,43 \\
76,19 \\
78,87 \\
74,11 \\
75,37\end{array}$ & $\begin{array}{l}69,05 \\
71,43 \\
70,24 \\
75,89 \\
73,81 \\
69,84 \\
75,89 \\
73,21 \\
72,47\end{array}$ & $\begin{array}{l}71,43 \\
72,02 \\
64,88^{\mathrm{b}} \\
71,13^{\mathrm{b}} \\
71,13 \\
69,64 \\
71,43 \\
71,13 \\
70,35^{\mathrm{b}}\end{array}$ & $\begin{array}{l}74,11 \\
74,40 \\
76,49^{\mathrm{a}} \\
77,08^{\mathrm{ab}} \\
76,62 \\
76,19 \\
76,79 \\
72,32 \\
75,52^{\mathrm{ab}}\end{array}$ & $\begin{array}{l}72,92 \\
77,68 \\
77,98^{\mathrm{a}} \\
83,03^{\mathrm{a}} \\
79,17 \\
78,57 \\
79,76 \\
79,17 \\
78,54^{\mathrm{a}}\end{array}$ \\
\hline $\begin{array}{l}\text { Peso de ovos } \\
\text { Egg weight }\end{array}$ & & & g & & & \\
\hline $\begin{array}{l}1 \\
2 \\
3 \\
4 \\
5 \\
6 \\
7 \\
8 \\
\text { Total }\end{array}$ & $\begin{array}{l}61,93 \\
62,32 \\
61,82 \\
61,92 \\
61,29 \\
62,47 \\
62,47 \\
61,79 \\
61,98\end{array}$ & $\begin{array}{l}61,34 \\
61,82 \\
62,92 \\
61,48 \\
62,47 \\
62,17 \\
62,45 \\
61,83 \\
62,06\end{array}$ & $\begin{array}{l}61,88 \\
61,35 \\
61,00 \\
62,24 \\
61,54 \\
61,83 \\
61,62 \\
60,98 \\
61,60\end{array}$ & $\begin{array}{l}60,68 \\
60,31^{b} \\
59,28 \\
61,08 \\
61,04 \\
61,70 \\
60,76 \\
60,25 \\
60,64\end{array}$ & $\begin{array}{l}62,00 \\
62,36^{\text {ab }} \\
63,43 \\
62,17 \\
61,83 \\
61,88 \\
63,41 \\
61,27 \\
62,30\end{array}$ & $\begin{array}{l}62,47 \\
62,84^{\mathrm{a}} \\
63,03 \\
62,39 \\
62,44 \\
63,04 \\
62,36 \\
63,07 \\
62,70\end{array}$ \\
\hline
\end{tabular}

Médias seguidas de mesma letra em cada linha e em cada fator não diferem pelo teste de Tukey $(P>0,05)$.

Means in the same row followed by the same letter are not different by Tukey test $(P>05)$. 
FARIA et al.

associação entre os níveis de $0,24 \%$ de sódio e 0,35 e $0,55 \%$ de fósforo total.

As características massa de ovos e índice de conversão alimentar (Tabela 3) não sofreram efeito significativo dos níveis de sódio em todos os períodos experimentais. Por outro lado, a massa de ovos foi afetada negativamente no terceiro, quarto, sexto, sétimo e oitavo períodos, quando as galinhas foram alimentadas com o nível de $0,35 \%$ de P total, principalmente. No período experimental total, verificouse menor produção de massa de ovos com a utilização de $0,35 \%$ de fósforo total.

Em alguns estudos desenvolvidos com sódio, constatou-se comprometimento da produção de ovos e conversão alimentar, quando os níveis dietéticos empregados foram de 0,1\% (REID, 1977; CHOI et al., 1980; e DAMRON e HARMS, 1980), os quais não foram adotados no presente experimento. Por outro lado, com níveis mais elevados, há concordância com os resultados obtidos por REID (1977), LEESON e SUMMERS (1979), CHOI et al. (1980),
DAMROM e HARMS (1980), JUNQUEIRA et al. (1984) e JUNQUEIRA (1988).

JACKSON et al. (1987), embora não tenham observado interação entre os níveis de sódio e fósforo para a característica peso dos ovos, constataram redução do peso, quando elevaram o nível dietético de sódio de 0,15 para $0,45 \%$. No presente trabalho, houve interação dos fatores em quatro períodos, sendo que, em três deles, o peso dos ovos foi reduzido conforme o aumento de sódio na dieta $(0,24 \%$ de sódio com $0,55 \%$ de fósforo total).

$\mathrm{Na}$ análise global do experimento, o comprometimento do desempenho das poedeiras alimentadas com o nível de $0,35 \%$ de fósforo total é comparável aos resultados obtidos por SUMMERS et al. (1976), HOLDER (1981) e JUNQUEIRA (1988). Níveis de fósforo inferiores a $0,35 \%$ também prejudicaram o desempenho das aves (MILES et al., 1983; BAR e HURWITZ, 1984). Os resultados obtidos com os níveis de fósforo nas dietas coincidem com as recomendações de HARMS (1982) e ROLAND (1986).

Tabela 3 - Massa de ovos e índice de conversão alimentar, em cada período de sete dias e no período experimental total, de galinhas alimentadas com diferentes níveis dietéticos de sódio e fósforo

Table 3 - Egg mass and feed:gain ratio in each seven days period and in the total experimental period, of the hens fed different dietary levels of sodium and phosphorus

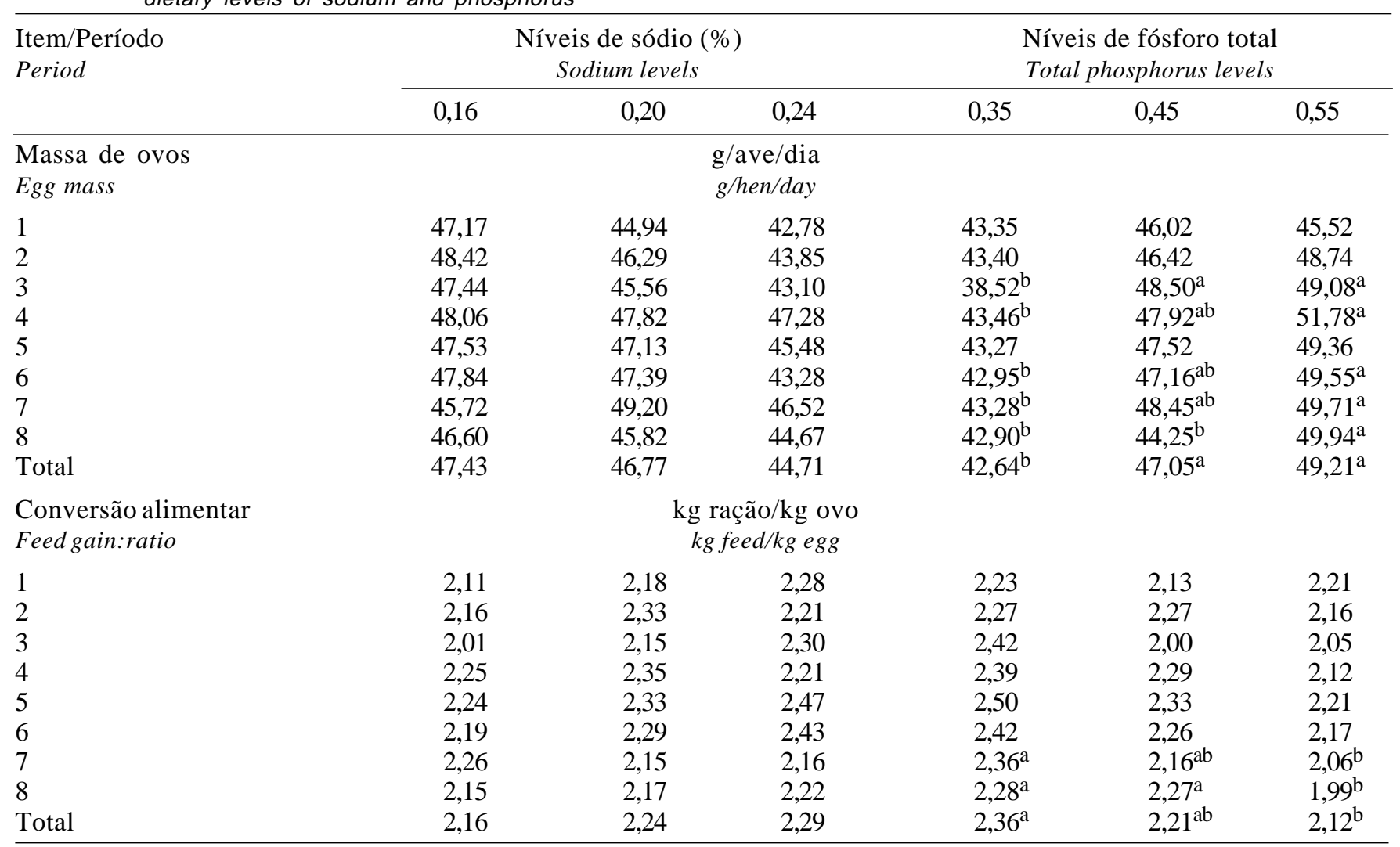

Médias seguidas de mesma letra em cada linha e em cada fator não diferem pelo teste de Tukey $(P>0,05)$.

Means in the same row followed by the same letter are not different by Tukey test $(P>05)$. 
464 Rev. bras. zootec.

Níveis de fósforo total ligeiramente abaixo de $0,45 \%$ resultaram em prejuízo do desempenho, segundo observações de VANDEPOPULIERE e LYONS (1992), que utilizaram o nível de $0,40 \%$ de fósforo total.

Os resultados das características que expressam a qualidade da casca dos ovos encontram-se na Tabela 4. Somente no sexto período experimental, a porcentagem de casca foi superior com a utilização do nível de $0,35 \%$ de fósforo total em relação ao nível intermediário. Nos demais períodos, a porcentagem de casca, espessura de casca e densidade aparente dos ovos não foram influenciadas pelos diferentes níveis de sódio e fósforo. Por outro lado, JUNQUEIRA (1988) constatou melhoria na densidade aparente dos ovos, com o emprego de $0,28 \%$ de sódio dietético, independente da fonte de sódio utilizada. A única ressalva seria o fato de que o nível máximo de sódio adotado no presente estudo foi de $0,24 \%$. Outra discordância ocorreu em relação aos achados de JUNQUEIRA et al. (1984), que detectaram maiores valores para a densidade aparente, quando administraram rações com $0,22 \%$ de sódio, embora essa ocorrência tenha sido registrada com a utilização de um nível de $1,4 \%$ de fósforo total.

Tabela 4 - Porcentagem de casca, espessura de casca e densidade aparente do ovo, em cada período de sete dias e no período experimental total, de galinhas alimentadas com diferentes níveis dietéticos de sódio e fósforo Table 4 - Shell percent, shell thickness and egg specific gravity in each seven days period and in the total experimental period, of the hens fed different dietary levels of sodium and phosphorus

\begin{tabular}{|c|c|c|c|c|c|c|}
\hline \multirow[t]{2}{*}{$\begin{array}{l}\text { Item/Período } \\
\text { Period }\end{array}$} & \multicolumn{3}{|c|}{$\begin{array}{l}\text { Níveis de sódio }(\%) \\
\text { Sodium levels }\end{array}$} & \multicolumn{3}{|c|}{$\begin{array}{l}\text { Níveis de fósforo total } \\
\text { Total phosphorus levels }\end{array}$} \\
\hline & 0,16 & 0,20 & 0,24 & 0,35 & 0,45 & 0,55 \\
\hline $\begin{array}{l}\text { Porcentagem de casca } \\
\text { Shellpercentage }\end{array}$ & & & $\%$ & & & \\
\hline $\begin{array}{l}1 \\
2 \\
3 \\
4 \\
5 \\
6 \\
7 \\
8 \\
\text { Total }\end{array}$ & $\begin{array}{l}8,57 \\
8,65 \\
9,07 \\
8,95 \\
8,82 \\
8,71 \\
8,91 \\
8,74 \\
8,80\end{array}$ & $\begin{array}{l}8,62 \\
8,84 \\
8,96 \\
8,93 \\
8,75 \\
8,78 \\
8,79 \\
8,71 \\
8,80\end{array}$ & $\begin{array}{l}8,76 \\
8,92 \\
9,13 \\
9,08 \\
9,01 \\
8,95 \\
8,92 \\
8,93 \\
8,96\end{array}$ & $\begin{array}{l}8,68 \\
8,70 \\
9,00 \\
9,01 \\
8,95 \\
8,98^{a} \\
8,90 \\
8,75 \\
8,87\end{array}$ & $\begin{array}{l}8,54 \\
8,70 \\
8,92 \\
8,96 \\
8,83 \\
8,60^{\mathrm{b}} \\
8,78 \\
8,79 \\
8,77\end{array}$ & $\begin{array}{l}8,74 \\
9,01 \\
9,24 \\
8,97 \\
8,79 \\
8,80^{\text {ab }} \\
8,94 \\
8,85 \\
8,92\end{array}$ \\
\hline $\begin{array}{l}\text { Espessura de casca } \\
\text { Shell thickness }\end{array}$ & & & $\mathrm{m}$ & & & \\
\hline $\begin{array}{l}1 \\
2 \\
3 \\
4 \\
5 \\
6 \\
7 \\
8 \\
\text { Total }\end{array}$ & $\begin{array}{l}0,34 \\
0,35 \\
0,35 \\
0,35 \\
0,34 \\
0,34 \\
0,35 \\
0,34 \\
0,34\end{array}$ & $\begin{array}{l}0,34 \\
0,34 \\
0,34 \\
0,34 \\
0,34 \\
0,34 \\
0,34 \\
0,33 \\
0,34\end{array}$ & $\begin{array}{l}0,35 \\
0,35 \\
0,35 \\
0,35 \\
0,35 \\
0,34 \\
0,35 \\
0,35 \\
0,35\end{array}$ & $\begin{array}{l}0,34 \\
0,34 \\
0,34 \\
0,35 \\
0,35 \\
0,34 \\
0,34 \\
0,33 \\
0,34\end{array}$ & $\begin{array}{l}0,34 \\
0,34 \\
0,35 \\
0,35 \\
0,34 \\
0,33 \\
0,34 \\
0,34 \\
0,34\end{array}$ & $\begin{array}{l}0,35 \\
0,35 \\
0,35 \\
0,35 \\
0,34 \\
0,34 \\
0,35 \\
0,35 \\
0,35\end{array}$ \\
\hline $\begin{array}{l}\text { Densidade aparente } \\
\text { Specific gravity }\end{array}$ & & & $\mathrm{H}_{2} \mathrm{O}$ & & & \\
\hline $\begin{array}{l}1 \\
2 \\
3 \\
4 \\
5 \\
6 \\
7 \\
8 \\
\text { Total }\end{array}$ & $\begin{array}{l}1,080 \\
1,082 \\
1,082 \\
1,082 \\
1,082 \\
1,081 \\
1,081 \\
1,081 \\
1,081\end{array}$ & $\begin{array}{l}1,081 \\
1,082 \\
1,082 \\
1,081 \\
1,081 \\
1,082 \\
1,080 \\
1,081 \\
1,081\end{array}$ & $\begin{array}{l}1,081 \\
1,083 \\
1,084 \\
1,083 \\
1,082 \\
1,082 \\
1,081 \\
1,082 \\
1,082\end{array}$ & $\begin{array}{l}1,081 \\
1,082 \\
1,083 \\
1,082 \\
1,082 \\
1,083 \\
1,081 \\
1,080 \\
1,082\end{array}$ & $\begin{array}{l}1,080 \\
1,082 \\
1,082 \\
1,082 \\
1,081 \\
1,080 \\
1,080 \\
1,081 \\
1,081\end{array}$ & $\begin{array}{l}1,081 \\
1,084 \\
1,084 \\
1,082 \\
1,081 \\
1,081 \\
1,081 \\
1,082 \\
1,082\end{array}$ \\
\hline
\end{tabular}

Médias seguidas de mesma letra em cada linha e em cada fator não diferem pelo teste de Tukey $(P>0,05)$.

Means in the same row followed by the same letter are not different by Tukey test $(P>05)$. 
FARIA et al.

Os resultados obtidos para o elemento fósforo, neste estudo, diferiram dos de JUNQUEIRA (1988), que constatou prejuízo da qualidade da casca com a administração de 0,30\% de fósforo total, e de HOLDER (1981) e LEESON et al. (1993), os quais verificaram melhoria da qualidade da casca com $0,40 \%$ de fósforo total ou, então, prejuízo com o nível de $0,45 \%$. No entanto, há concordância dos resultados com um dos experimentos desenvolvidos por VANDEPOPULIERE e LYONS (1992), que não encontraram diferenças nos valores de densidade aparente dos ovos produzidos por galinhas alimentadas com dietas contendo 0,40 ou $0,50 \%$ de fósforo total.

Considerando a variabilidade dos resultados para as características de qualidade de casca dos ovos com a administração de diferentes níveis de fósforo, principalmente baixos níveis, acredita-se que as colocações realizadas por ROLAND e GORDON (1996) sejam oportunas, uma vez que ocorre grande variação entre as aves em relação aos efeitos de dietas com níveis marginais de fósforo, além de a melhoria da qualidade da casca possuir caráter temporário.

Os resultados referentes às características sangüíneas encontram-se na Tabela $5 . \mathrm{A} \mathrm{pO}_{2}$ apresentou maior valor quando da utilização do nível de $0,20 \%$ de sódio comparado ao nível de $0,24 \%$. A concentração de leucócitos foi maior para as galinhas que receberam dietas com $0,35 \%$ de fósforo em relação ao nível de $0,55 \%$. A concentração de hemoglobina centrifugada variou entre os níveis de fósforo, sendo o menor valor apresentado quando as galinhas receberam a ração contendo o nível de $0,55 \%$ em relação ao nível de $0,45 \%$ de fósforo. Para a característica hemácias, houve efeito de blocos, no qual as aves mais pesadas apresentaram maior concentração dessas células sangüíneas (não-mostrado).

JUNQUEIRA (1988) encontrou variações significativas nos valores de $\mathrm{pH}$ entre os níveis de sódio testados, dentro da mesma fonte (cloreto de sódio), resultado não-detectado nesse experimento. $\mathrm{O}$ mesmo modelo de resposta também foi verificado para a $\mathrm{pCO}_{2}$.

Dos trabalhos revisados, nenhum deles realizou a análise hematimétrica. No entanto, os valores obtidos para a concentração de hemácias e hemoglobina centrifugada estão dentro dos valores normais sugeridos por ZINKL (1986), diferindo somente em relação aos leucócitos que se mantiveram com valores inferiores (7467 a 8133 células/ $\mathrm{mm}^{3}$ ) contra uma média sugerida de 12.000 células/ $\mathrm{mm}^{3}$. Os valores encontrados para o hematócrito são comparáveis aos propostos por MACARI et al. (1994). Os níveis de fósforo utilizados no presente experimento não alteraram os indicadores sangüíneos do equilíbrio ácido-base $\left(\mathrm{pH}, \mathrm{pCO}_{2}\right)$, o que provavelmente ocorreria se níveis mais elevados de fósforo fossem administrados às poedeiras, como constatado por MILES et al. (1983), os quais utilizaram 0,70 e $1,50 \%$ de fósforo total.

Tabela 5 - Valores médios de $\mathrm{pH}, \mathrm{pO}_{2}, \mathrm{pCO}_{2}$, hemácias, leucócitos, hemoglobina, hemoglobina centrifugada e hematócrito de galinhas alimentadas com diferentes níveis dietéticos de sódio e fósforo

Table 5 - Means of $\mathrm{pH}, \mathrm{pO}_{2} \mathrm{pCO}_{2}$, erythrocytes, leucocytes, haemaglobin, centrifugate haemoglobin and haematocrit of the hens fed different dietary levels of sodium and phosphorus

\begin{tabular}{|c|c|c|c|c|c|c|}
\hline \multirow[t]{2}{*}{$\begin{array}{l}\text { Item/Período } \\
\text { Period }\end{array}$} & \multicolumn{3}{|c|}{$\begin{array}{l}\text { Níveis de sódio (\%) } \\
\text { Sodium levels }\end{array}$} & \multicolumn{3}{|c|}{$\begin{array}{l}\text { Níveis de fósforo total } \\
\text { Total phosphorus levels }\end{array}$} \\
\hline & 0,16 & 0,20 & 0,24 & 0,35 & 0,45 & 0,55 \\
\hline $\mathrm{pH}$ & 7,35 & 7,37 & 7,36 & 7,39 & 7,35 & 7,33 \\
\hline $\mathrm{pO}_{2}, \mathrm{mmHg}$ & $85,42^{\mathrm{ab}}$ & $97,33^{\mathrm{a}}$ & $80,75^{\mathrm{b}}$ & 93,58 & 82,17 & 87,75 \\
\hline $\mathrm{pCO}_{2}, \mathrm{mmHg}$ & 30,50 & 27,58 & 29,17 & 25,25 & 32,25 & 29,75 \\
\hline Hemácias, cel $/ \mathrm{mm}^{3}$ & 2325 & 2270 & 2211 & 2296 & 2237 & 2273 \\
\hline $\begin{array}{l}\text { Erythrocytes } \\
\text { Leucócitos, cel } / \mathrm{mm}^{3} \\
\text { Leucocytes }\end{array}$ & 8133 & 8450 & 7467 & $9483^{\mathrm{a}}$ & $7717^{\mathrm{ab}}$ & $6850^{\mathrm{b}}$ \\
\hline $\begin{array}{l}\text { Hemoglobina }(\mathrm{Hb}), \mathrm{g} / 100 \mathrm{~mL} \\
\text { Haemoglobin }\end{array}$ & 13,59 & 13,45 & 13,60 & 13,76 & 13,21 & 13,67 \\
\hline $\begin{array}{l}\text { Hb centrifugada, } \mathrm{g} / 100 \mathrm{~mL} \\
\text { Hb centrifugated }\end{array}$ & 9,02 & 8,35 & 8,69 & $8,68^{\mathrm{ab}}$ & $8,23^{\mathrm{b}}$ & $9,14^{\mathrm{a}}$ \\
\hline $\begin{array}{l}\text { Hematócrito, \% } \\
\text { Haematocrit }\end{array}$ & 26,08 & 26,25 & 26,75 & 27,00 & 26,17 & 25,92 \\
\hline
\end{tabular}

Médias seguidas de mesma letra em cada linha e em cada fator não diferem pelo teste de Tukey $(P>0,05)$

Means in the same row followed by the same letter are not different by Tukey test $(P>.05)$. 


\section{Conclusões}

O desempenho das poedeiras foi comprometido quando alimentadas com as dietas contendo $0,35 \%$ de fósforo total sem benefícios para as características de qualidade de casca dos ovos. Embora o nível de $0,45 \%$ de fósforo tenha proporcionado taxas normais de produção pelas poedeiras, seria conveniente considerar o período de tempo de sua utilização para efetiva avaliação de seus efeitos.

Níveis de sódio mais elevados que $0,16 \%$ não se mostraram vantajosos para as características de desempenho e qualidade da casca dos ovos, considerando o padrão de consumo de ração e os níveis de fósforo adotados no presente experimento.

As variáveis que expressam o equilíbrio ácidobase das poedeiras não foram alteradas em função da utilização de diferentes níveis dietéticos de sódio e fósforo total.

\section{Referências Bibliográficas}

BAR, A., HURWITZ, S. 1984. Eggshell quality, medullary bone ash, intestinal calcium and phosphorus absorption and calcium-binding protein in phosphorus-deficient hens. Poult. Sci., 63(10):1975-1979.

CHOI, J.H., MILES, R.D., HARMS, R.H. 1980. Interaction of dietary phosphorus and sodium chloride levels on blood phosphorus and egg production of hens. Poult. Sci., 59(8):1897-1900.

DAMRON, B.L., HARMS, R.H. 1980. Interaction of dietary salt, calcium, and phosphorus levels for laying hens. Poult. Sci., 59(1):82-85.

HARMS, R.H. 1982. The influence of nutrition on eggshell quality. Part 2: Phosphorus. Feedstuffs, 54(19):25-26.

HOLDER, D.P. 1981. Dietary phosphorus requirements of force-molted leghorn hens. Poult. Sci., 60(2):433-437.

JACKSON, M.E., HELLWIG, H.M., WALDROUP, P.W. 1987. Shell quality: potential for improvement by dietary means and relationhips with egg size. Poult. Sci., 66(10):17021713.

JAIN, N.C. 1986. Hematologic techniques. In:_____. Schalm's veterinary hematology. Philadelphia: Lea \& Febiger, 4.ed. p.20-86.

JUNQUEIRA, O.M. Contribuição para o estudo do metabolismo de alguns minerais nas galinhas poedeiras. Jaboticabal, SP: FCAVJ, 1988. 101p. Tese (Livre-Docência) - Universidade Estadual Paulista, 1988.

JUNQUEIRA, O.M., MILES, R.D. HARMS, R.H. 1984. Interrelationship between phosphorus, sodium, and chloride in the diet of laying hens. Poul. Sci., 63(6):1229-1236.

LEESON, S., SUMMERS, J.D. 1991. Commercial poultry nutrition. Guelph: University Books, 238p.

LEESON, S., SUMMERS, J.D. 1979. Dietary salt for layers. Nutr. Rep. Int., 19(2):173-178.
LEESON, S., SUMMERS, J.D., CASTON, L. 1993. Response of brown-egg strain layers to dietary calcium or phosphorus. Poult. Sci., 72(8):1510-1514.

MACARI, M., FURLAN, R.L., GONZALES, E. 1994. Fisiologia aviária aplicada a frangos de corte. Jaboticabal: FUNEP. 296p.

MILES, R.D., COSTA, P.T., HARMS, R.H. 1983. The influence of dietary phosphorus level on laying hen performance, eggshell quality, and various blood parameters. Poult. Sci., 62(6):1033-1037.

MORENG, R.E., AVENS, J.S. 1990. Ciência e produção de aves. São Paulo: Roca. 380p.

NATIONAL RESEARCH COUNCIL - NRC. 1994. Nutrient requirements of poultry. 9.ed. Washington: National Academy Science. $155 \mathrm{p}$.

NATIONAL RESEARCH COUNCIL - NRC. 1984. Nutrient requirements of poultry. 8.ed. Washington: National Academy Science. $71 \mathrm{p}$.

OUSTERHOUT, L.E. 1980. Effects of calcium and phosphorus levels on egg weight and egg shell quality in laying hens. Poult. Sci., 59(7):1480-1484.

REID, B.L. 1977. Dietary sodium for laying hens. Poult. Sci., 56(1):373-374.

ROLAND, D.A. 1986. Eggshell quality. III. Calcium and phosphorus requirements of commercial leghorns. World's Poult. Sci. J., 42(2):154-165.

ROLAND, D.A., GORDON, R. 1996. Phytase helps optimize phosphorus, calcium in layer diets. Feedstuffs, 68(10):16,17,37,39.

ROSTAGNO, H.S., SILVA, D.J., COSTA, P.M.A. et al. 1985. Composição de alimentos e exigências nutricionais de aves e suínos (Tabelas Brasileiras). Viçosa: Imprensa Universitária. $59 \mathrm{p}$.

SAID, N.W., SULLIVAN, T.W. 1985. A comparison of continous and phased levels of dietary phosphorus for commercial laying hens. Poult. Sci., 64(9):1763-1771.

SAKOMURA, N.K., GARCIA, J.R.M., ARIKI, J. et al. 1993. Fontes e níveis de fósforo em rações de poedeiras comerciais. Ars Vet., 9(1):54-66.

SAS INSTITUTE (Cary, USA). SAS language reference. Cary, 1988. 530p.

SCHEIDELER, S.A., AL-BATSHAN, H. 1994. Basics of calcium, phosphorus nutrition in layers styudied. Feedstuffs, 66(14):15-16.

SCOTT, M.L., NESHEIM, M.D., YOUNG, R.J. 1982. Nutrition of the chicken. Ithaca: M.L. Scott \& Associates. 562p.

SUMMERS, J.D, GRANDHI, R., LEESON, S. 1976. Calcium and phosphorus requirements of the laying hen. Poult. Sci., 56(1):402-413.

VANDEPOPULIERE, J.M., LYONS, J.J. 1992. Effect of inorganic phosphate source and dietary phosphorus level on laying hen performance and eggshell quality. Poult. Sci., 71(6): 1022-1031.

ZINKL, J.G. 1986. Avian hematology. In: JAIN, N.C. (Ed.) Schalm's veterinary hematology. Philadelphia: Lea \& Febiger, 4.ed. p.256-273.

Recebido em: 22/03/99

Aceito em: 27/09/99 